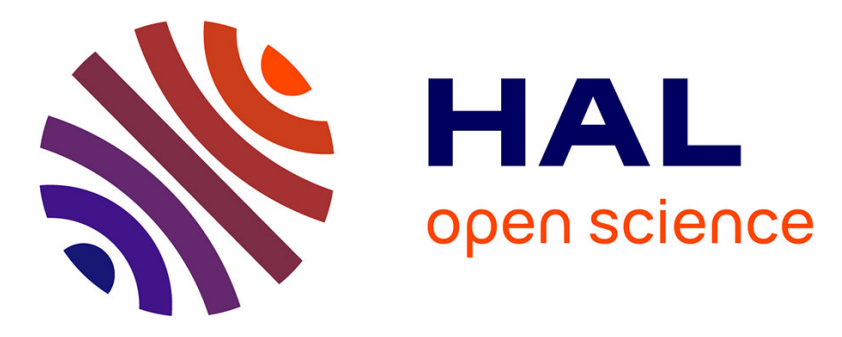

\title{
A van der Waals force-based adhesion model for micromanipulation.
}

Sébastien Alvo, Pierre Lambert, Michaël Gauthier, Stéphane Régnier

\section{To cite this version:}

Sébastien Alvo, Pierre Lambert, Michaël Gauthier, Stéphane Régnier. A van der Waals force-based adhesion model for micromanipulation.. Journal of Adhesion Science and Technology, 2010, 24 (15-16), pp.2415-2428. 10.1163/0169942410X508334 . hal-00545519

\section{HAL Id: hal-00545519 https://hal.science/hal-00545519}

Submitted on 10 Dec 2010

HAL is a multi-disciplinary open access archive for the deposit and dissemination of scientific research documents, whether they are published or not. The documents may come from teaching and research institutions in France or abroad, or from public or private research centers.
L'archive ouverte pluridisciplinaire HAL, est destinée au dépôt et à la diffusion de documents scientifiques de niveau recherche, publiés ou non, émanant des établissements d'enseignement et de recherche français ou étrangers, des laboratoires publics ou privés. 


\title{
A van der Waals Force-Based Adhesion Model for Micromanipulation
}

\author{
S. Alvo ${ }^{a b *}$, P. Lambert ${ }^{b c}$, M. Gauthier ${ }^{b}$ and S. Régnier ${ }^{a}$ \\ ${ }^{a}$ Institut des Systèmes Intelligents et Robotique (ISIR), \\ Université Pierre et Marie Curie - Paris 6, UMR CNRS 7222, \\ 4 place Jussieu, Boite Courrier 173, \\ 75252 Paris Cedex 05, France. \\ ${ }^{b}$ FEMTO-ST Institute, Dept. AS2M, UMR CNRS 6174 - UFC / ENSMM / UTBM, \\ 24 rue Alain Savary, 25000 Besançon, France. \\ ${ }^{c}$ Service des Systèmes Bio-, électromécaniques (BEAMS) CP 165/56, \\ Université libre de Bruxelles, \\ 50 Avenue Roosevelt, \\ B - 1050 Bruxelles, Belgium.
}

\begin{abstract}
The robotic manipulation of microscopic objects is disturbed directly by the adhesion between the end-effector and the objects. In the micro scale, no reliable model of adhesion is available and currently the behaviour of the micro-objects cannot be predicted before experiments. This paper proposes a new model of adhesion based on the analytical resolution of the coupling between the mechanical deformation of the micro-objects and van der Waals forces. In the nanoscale, the impact of the deformation can be neglected and the proposed model is thus similar to the classical expression for van der Waals forces. In the microscale, the deformation induces van der Waals forces to increase significantly and a new analytical expression is proposed. The limit of validity of this 'deformable van der Waals forces' is also discussed. This result can be used as an alternative to classical adhesion-deformation models in literature (Johnson-Kendall-Roberts (JKR) or DerjaguinMuller-Toporov (DMT)), which have been validated at the macroscale but are not sufficient to describ the interaction forces in the microscale (typically from $100 \mathrm{~nm}$ to $500 \mu \mathrm{m}$ ).
\end{abstract}

keywords : pull off force, deformation, adhesion, van der Waals forces, micromanipulation

\section{Introduction}

The study of micromanipulation consists of developing models and fabricating experimental tools for the individual manipulation and characterization of microcomponents. The manipulator covers a large variety of microgrippers (mechanical and optical tweezers, capillary grippers...) actuated using numerous physical effects (thermal expansion, piezoelectricity, smart memory alloy...). Characterization mainly implies mechanical characterization of stiffness performed, for example, with an atomic force microscope. The major industrial perspective of micromanipulation is to develop reliable micro-assembly techniques, based on robotic assembly or self-assembly. Both require adequate models to estimate the surface forces disturbing the micromanipulation.

The goal of developing models at the microscale may be questioned for many reasons:

1. the task is huge and the forces dominating at the micro- and nanoscales can be modeled only partially: for example, some of them cannot be modeled in a quantitative way (e.g. hydrogen bonds) suitable for robotics purpose, most of proposed models are valid only at equilibrium (at least all the models based on the derivation of surface or potential energies);

2. it is impossible sometimes to know the parameters involved in the existing models, for example, the distribution of electrical charges on a dielectric oxide layer;

3. in the micro- and nanoscales, the physical measurements suffer from a very large experimental dispersion, which makes the models' refinements questionable. According to individual's experience, experimental measurements are typically obtained with an error greater than a few tens of percent.

${ }^{*}$ To whom correspondence should be addressed. Tel.: + 33(0)1 442763 79; e-mail: sebastien.alvo@isir.upmc.fr 
Nevertheless the use of - even basic - models helps the microrobotician to roughly describe the micro-objects behavior and design best grippers and tools. Classical adhesion models like JKR [1], DMT [2] and MaugisDugdale [3] are usually proposed to study adhesion in micromanipulation [4]. These models are based on the elastic deformation of two solids in contact (e.g. microcomponent/gripper in micromanipulation) and cannot match the experimental data very well at the microscale.

We propose, therefore, a new analytical model taking into account the elastic deformation and the van der Waals forces as the main source of adhesion [5,6]. Capillary condensation is supposed to play a major role in humid environments [7], but as a preliminary step in this paper, we will only consider dry environments in which capillary condensation can be neglected. Moreover, materials used are supposed to be non-conductive and neutral so electrostatic forces are not considered.

This paper is organized in the following sections. Section 2 presents the coupled problem of adhesion and deformation and the innovative iterative scheme used to solve it. Section 3 recalls the basics of van der Waals $(\mathrm{vdW})$ forces and presents the vdW interaction between a truncated sphere and a plane, which is used in the iterative resolution. Section 4 describes the iterative scheme which provides an implicit expression for the force. Two analytical approximations are also provided which are valid in nano- and microscales, respectively. Finally, Section 5 suggests further work and conclusions are drawn in Section 6.

\section{Problem description}

\subsection{The lack of adhesion model for micromanipulation}

The main idea of this paper is to extend the existing model to the microscale. On the one hand, JKR, DMT or Maugis models are usually used to compute pull-off forces (force needed to split two objects in contact). They are computed with global energy calculation and their efficiency is well established at macroscale [8] but they are not correlated with experimental microscale measurements. These models take into account the impact of the deformation on the pull-off force but they are restricted to sphere/plane contact and cannot be applied to more complex geometries (e.g parallelepipedic objects) usually used in microhandling [9].

On the other hand, atomic models based on the Hamaker computation could be extended from nanoscale to microscale. So van der Waals theory could be used to predict adhesion phenomena [6] for any kind of geometry (see Section 3.1). However, in the microscale, this theory underestimates pull-off forces. Moreover, it does not take into account the deformation which has a significant impact in microscale.

An Atomic Force Microscope (AFM) with a cantilever whose stiffness is $0.3 \mathrm{~N} / \mathrm{m}$ has been used to measure pull-off forces. Measurements have been carried out in a temperature and humidity controlled environment to minimize the capillary condensation. For example, the measured pull-off force is $1.6 \pm 0.6 \mu \mathrm{N}$ when a glass sphere with $10 \mu \mathrm{m}$ diameter contacts a glass substrate. JKR and DMT theories predict a larger force of $8.0 \mu \mathrm{N}$ and $10.7 \mu \mathrm{N}$ and the van der Waals theory predicts a smaller value of $0.6 \mu \mathrm{N}$. New investigation should be performed in order to predict pull-off forces better in the microscale.

So new models are necessary to understand the behavior of objects whose size is between $100 \mathrm{~nm}$ and $100 \mu \mathrm{m}$. This paper proposes a way to take into account the deformation of the object in the calculation of van der Waals forces.

\subsection{A new method for adhesion modeling}

The sum of van der Waals forces applied by an object to another one depends on their contact surface and can also be considered as a global force which induces a deformation. This deformation increases the contact surface and the global van der Waals forces too. This coupled problem can be seen as an algorithm that uses sequentially two models (Fig. 1). The first one computes van der Waals forces according to the object shape. The other one computes deformation shape according to an external load. An iterative calculation is able to converge to the physical equilibrium.

This generic principle can be applied to calculate or simulate adhesion forces of a large variety of geometries. However, this paper focuses on the analytical expression applied to sphere/plane contact based on an analytical expression for the van der Waals forces and a deformation model via the Hertz theory. For more complex geometries including roughness [10], the calculation of the van der Waals forces should be replaced by a numerical calculation, and another deformation model should be considered (e.g [11] for axisymmetric shapes) or modelled via finite element method.

\section{Modeling adhesion with van der Waals forces for simple rigid geometries}

The first step in the calculation is to calculate the van der Waals forces on a deformed object. A pairwise summation of the energy given by the Lennard-Jones potential leads to classical expression for the van der Waals 


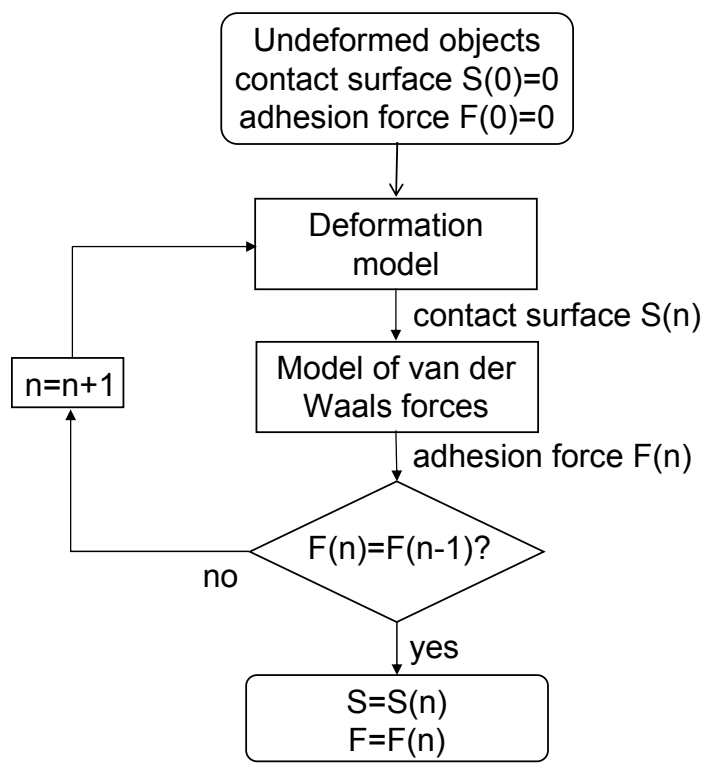

Figure 1: Algorithm proposed for calculating the adhesion force between two objects using the coupling between deformation and van der Waals forces.

forces. Computation for simple shape objects attracted by a plane is carried out here as an example which can be applied to any kind of geometries.

\subsection{The Lennard-Jones potential and the Hamaker approximation}

The Lifshitz theory considers "dance of charges" coupled with relativistic effects [12] to calculate electrodynamic free energy that is at the origin of van der Waals forces. It is commonly approximated using the Lennard-Jones potential:

$$
\phi(r)=\epsilon_{0}\left(\frac{r_{0}}{r}\right)^{12}-2 \epsilon_{0}\left(\frac{r_{0}}{r}\right)^{6} .
$$

This potential represents the interaction between two neutral atoms (or molecules) separated by a distance $r$ (Fig. 2). $r_{0}$ is the equilibrium distance when the force $F=-\partial \phi / \partial r$ (Fig. 3) is null and $\epsilon_{0}$ is the corresponding energy. The $1 / r^{12}$ term describes the strong repulsion that appears when the two atoms are closer than $r_{0}$. For $r>r_{0}$, the Lennard-Jones potential is usually approximated by the $1 / r^{6}$ term (Fig. 2):

$$
\phi(r)=-\frac{C}{r^{6}} \quad \text { where } \quad C=2 \epsilon_{0} r_{0}{ }^{6} .
$$

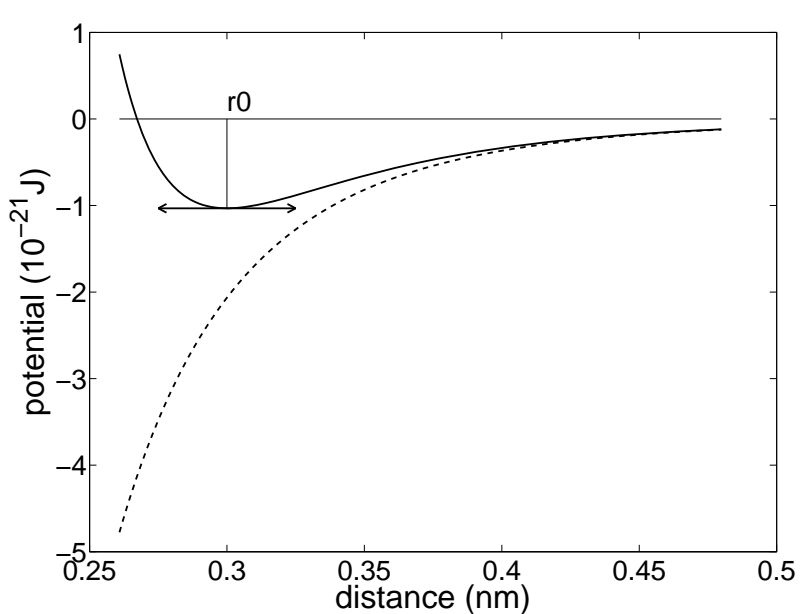

Figure 2: Comparison of Lennard Jones potential (solid line) and $1 / r^{6}$ potential (dashed line).

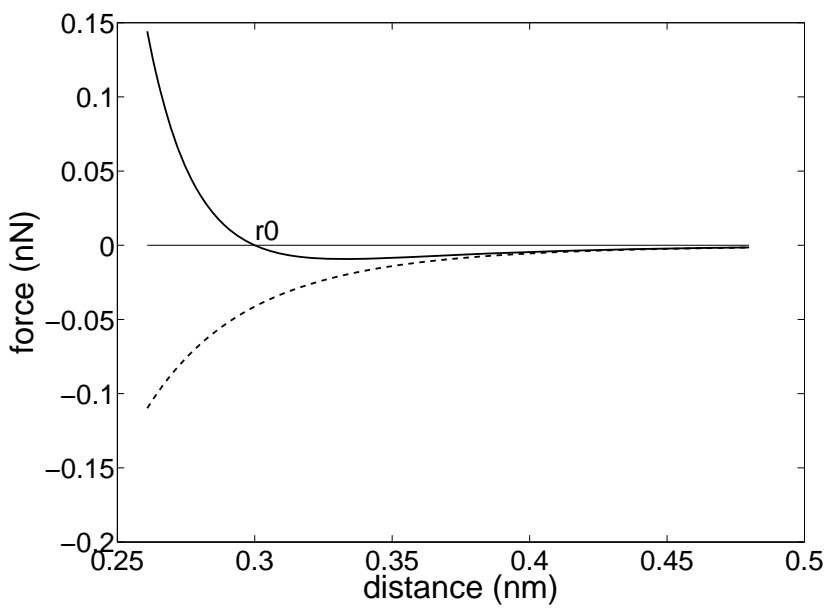

Figure 3: Comparison of forces derived from the Lennard Jones potential (solid line) and from the $1 / r^{6}$ potential (dashed line).

Adhesion forces are computed by a pairwise summation of the Lennard-Jones energies. Considering molecular densities of materials, the discrete summation can be replaced with an integral. This method is commonly called the Hamaker approximation [13] which is summarized very well in [14]. 


\subsection{Interaction between a molecule and an infinite plane}

The first step in computation of the van der Waals forces is to sum every pairwise energy between the plane and a molecule of the second object. The van der Waals forces have a short cut-off radius so the plane can be considered as infinite without any influence on the result. Then, using notations of Fig. 4 and considering a molecular density $\beta_{1}$ for the plane $\Omega_{1}$, the adhesion energy $\pi_{p}$ between a molecule and a plane can be formulated as below:

$$
\pi_{p}(z)=\beta_{1} \int_{\Omega_{1}} \frac{-C}{r^{6}} d \Omega_{1}=-\frac{\pi C \beta_{1}}{6 z^{3}} .
$$

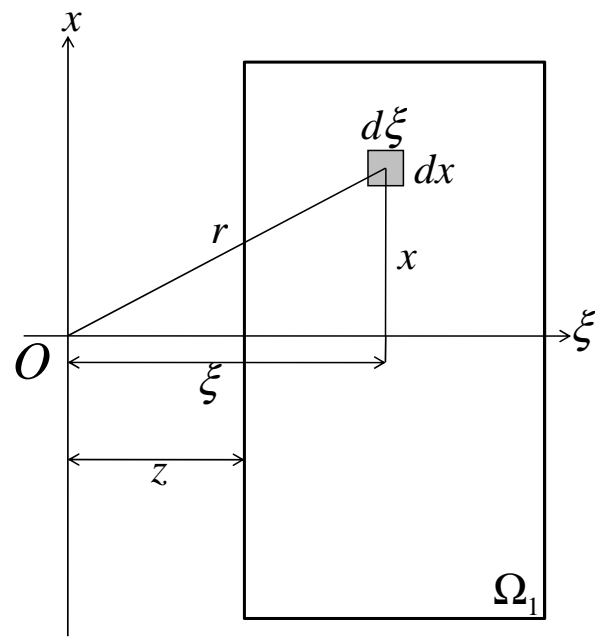

Figure 4: Notations used for the first step of van der Waals forces calculation: attraction force between a molecule and a rigid, infinite plane.

\subsection{Interaction between a sphere and an infinite plane}

Now the second part of the calculation can be performed by considering a molecular density $\beta_{2}$ for the second object (a sphere of radius R). For sphere and plane, the energy of interaction is:

$$
\Pi_{s p}=\beta_{1} \beta_{2} \int_{\Omega_{2}}-\frac{\pi C}{6 z^{3}} d \Omega_{2} .
$$

The Hamaker constant $A=\pi^{2} C \beta_{1} \beta_{2}$ appears in the formulation.

The sphere studied is supposed to be larger than its distance from the plane (assumed to be $r_{0}=0.3 \mathrm{~nm}$ ). So for $R>>r_{0}$ the force that derive from the potential of eq. (4) is:

$$
F_{s p}=-\frac{A R}{6 r_{0}^{2}}
$$

\subsection{Interaction between a truncated sphere and an infinite plane}

We consider a truncated sphere which represents the volume of a sphere of radius $R$ where the cap with a $\delta$ height has been removed (see Fig. 5). The planar surface of the truncated sphere is at a distance $r_{0}$ from the plane. Integral of eq. (4) has now to be computed with a modified $\Omega_{2}$ described in Fig. 5. The volume $\Omega_{2}$ extends from $\xi=\delta$ to $2 R$ for the truncated sphere rather than from 0 for the full sphere. Thus, still considering non-atomic sized spheres $\left(R>>r_{0}\right)$ and little deformations $(R>>\delta)$, a new expression for the van der Waals forces can be formulated as:

$$
F_{s^{\prime} p}=-\frac{A R\left(r_{0}+2 \delta\right)}{6 r_{0}^{3}} \text {. }
$$

The first term of this force is equal to the force between a full $R$-radius sphere and a plane seen in eq. (5). Considering that $\delta<<R$, the term $\frac{A R \delta}{3 r_{0}^{3}}$ represents the force due to a cylinder whose radius corresponds to the truncated part of the sphere. The increase of the contact surface between a sphere and a truncated sphere induces an increase of the van der Waals forces despite the reduction of the volume.

The force computed in eq. (6) is used in our coupled model to represent the van der Waals forces applied to a deformed object. The van der Waals forces computation consequently considers that the plane is rigid. 


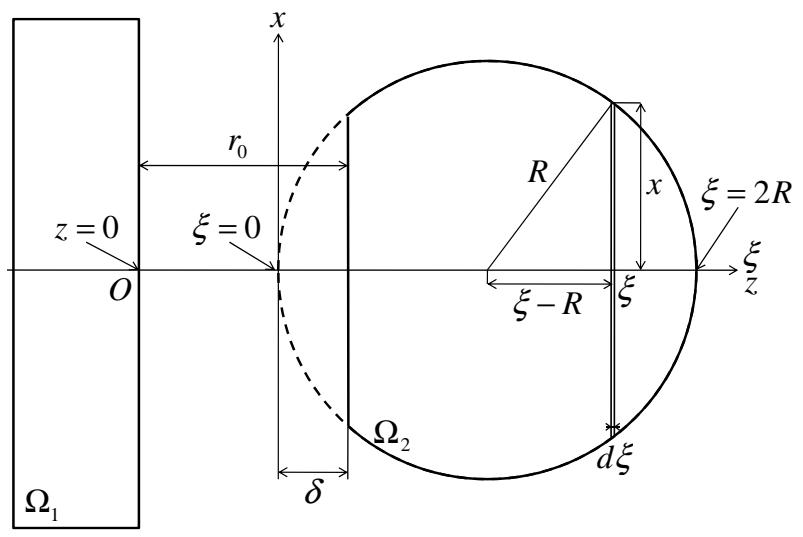

Figure 5: Integration of van der Waals forces between a truncated sphere and an infinite plane.

\section{Sphere deformation under van der Waals forces}

A deformation model is needed for the second phase of the algorithm presented in Fig. 1. In the case of a contact between a sphere and a plane, deformation according to load can be computed via the Hertz theory.

\subsection{Hertz contact modeling}

This model which defines the contact surface is the basis for the other models well known in adhesion modeling (JKR and DMT). It computes the radius of contact $a$ and the penetration $\delta$ for two spheres pressed together with a force $F$ (Fig. 6). Hertz model does not consider adhesion directly. This model is based on a geometrical and mechanical analysis under three assumptions:

- contact radius $a$ is small compared to the radii of spheres;

- there is no friction at the interface;

- there is no tensile stress in the contact area.

Elastic properties of the $i^{\text {th }}$ material are $E_{i}$ (Young's modulus) and $\nu_{i}$ (Poisson's coefficient). The modified Young's modulus $E^{*}$ is defined as:

$$
\frac{1}{E^{*}}=\frac{1-\nu_{1}^{2}}{E_{1}}+\frac{1-\nu_{2}^{2}}{E_{2}} .
$$

So in case of a sphere/plane contact, the contact radius $a$ and penetration depth $\delta$ can be calculated via Hertz theory:

$$
\begin{aligned}
a^{3} & =-\frac{3}{4} \frac{R F}{E^{*}}, \\
\delta & =\frac{a^{2}}{R} .
\end{aligned}
$$

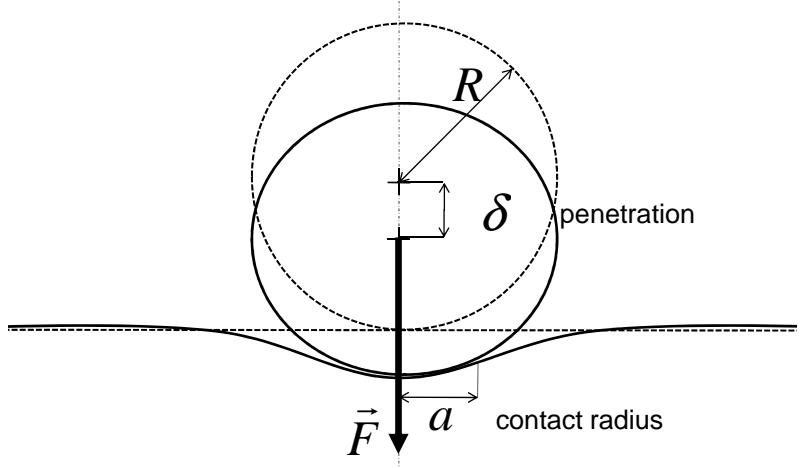

Figure 6: Sketch of a sphere/plane contact. 


\subsection{Computation of a model including deformation for a sphere/plane contact}

We assume that the deformed sphere can be seen as a truncated sphere which is described in Section 3.4. At every step of the algorithm, van der Waals forces $F_{n}$ are calculated by eq. (6) and the contact radius $a_{n}$ is given by the Hertz theory (eq. (8)). The penetration into the plane $\delta_{n}$ is linked to the contact radius $a_{n}$ by eq. (9). So eq. (6) can be rewritten as:

$$
F_{n}=-\frac{A\left(r_{0} R+2 a_{n}^{2}\right)}{6 r_{0}^{3}} .
$$

At the initial step of the algorithm, the contact radius is considered as null, i.e. $a_{0}=0$. For all steps $n$, eq. (8) implies:

$$
a_{n+1}=-\left(\frac{3 R}{4 E^{*}}\right)^{1 / 3} F_{n}{ }^{1 / 3}
$$

Considering eq. (10), a sequence $\left(a_{n}\right)$ can be defined:

$$
a_{n+1}=\lambda^{1 / 3}\left(r_{0} R+2 a_{n}^{2}\right)^{1 / 3},
$$

where

$$
\lambda=A R / 8 r_{0}^{3} E^{*} .
$$

The sequence (12) converges to its unique positive fixed point $a_{\infty}$. Fixed point is classically defined by $a_{n}=$ $a_{n+1}=a_{\infty}$, so $a_{\infty}$ is the unique real solution of eq. (14):

$$
a_{\infty}^{3}-2 \lambda a_{\infty}^{2}-\lambda r_{0} R=0 .
$$

This third-order equation can be solved analytically with the Cardan formula but the result is too complex to be exploited easily. The surface radius $a_{d v d W}$ of the deformable van der Waals (dvdW) model can be obtained by numerical computation of eq. (14) (see Fig. 7). Moreover, Sections 4.3 and 4.4 will show that an analytical solution exists for nano and microspheres.

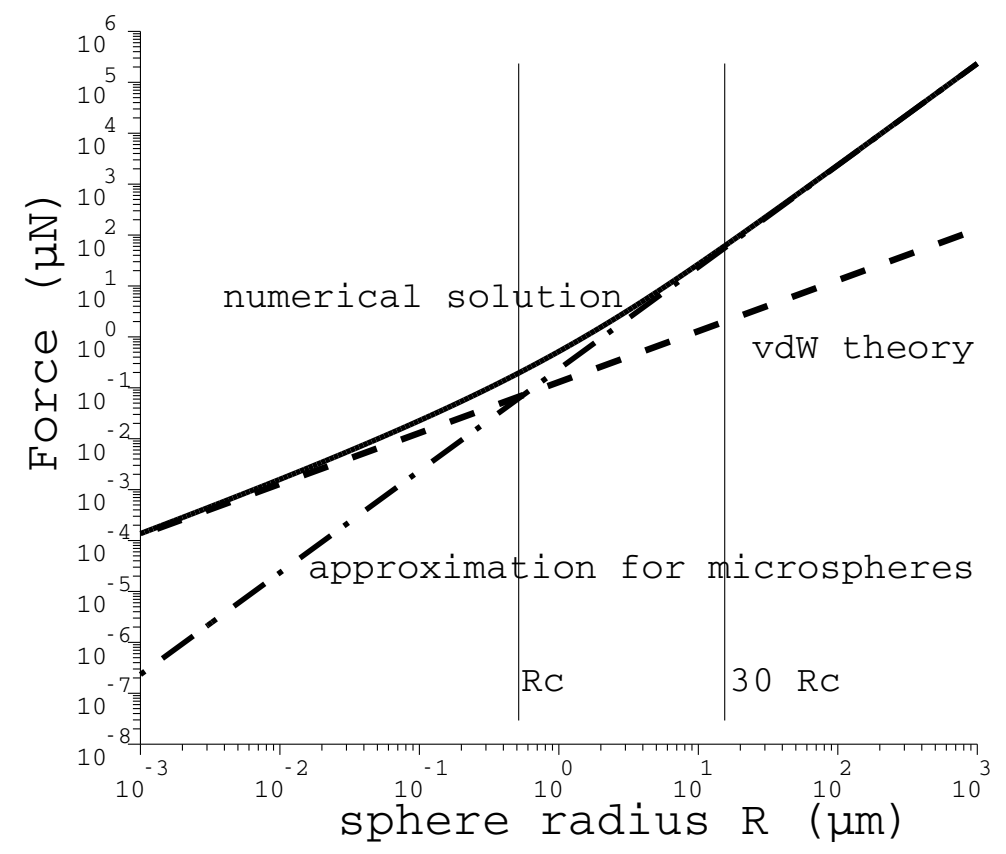

Figure 7: Comparison of forces computed with classical and deformable van der Waals theories at nano and microscales (log scale). Dashed line: classical van der Waals theory. Dash-dotted line: approximation of microspheres (eq. 18). Solid line: numerical solution of the deformable van der Waals (dvdW) model (eq. 14) matches classical van der Waals (eq. 5) at nanoscale and analytical formula (18) at microscale.

\subsection{Approximation of micro-sized spheres}

This subsection presents an expression for the van der Waals forces in the case of micro-sized spheres. Equation (14) could be normalized by the $(2 \lambda)^{3}$ term:

$$
\left(\frac{a_{\infty}}{2 \lambda}\right)^{3}-\left(\frac{a_{\infty}}{2 \lambda}\right)^{2}-\frac{R_{c}}{R}=0,
$$


where

$$
R_{c}=\frac{r_{0} R^{2}}{8 \lambda^{2}}=\frac{8 r_{0}^{7} E^{* 2}}{A^{2}}
$$

is a constant which depends only on properties of the sphere material.

If $R_{c} / R<<\left(a_{\infty} / 2 \lambda\right)^{3}$, eq. (15) can be rewritten as:

$$
\frac{a_{\infty}}{2 \lambda} \approx 1
$$

So for $R>>R_{c}$, the algorithm for the deformation caused by van der Waals forces converges to a contact radius:

$$
\tilde{a}_{d v d W}=2 \lambda=\frac{A R}{4 r_{0}^{3} E^{*}} .
$$

Applying this approximation to eq. (10), a simplified force can be solved out as below:

$$
\tilde{F}_{d v d W}=-\frac{A^{3} R^{2}}{48 r_{0}^{9} E^{* 2}} .
$$

For example, in case of glass objects (see Table 1$), R_{c}$ is $0.5 \mu \mathrm{m}$ and this approximation $\left(R>>R_{c}\right)$ can be used for glass micro-sized spheres commonly used in micromanipulation.

Table 1: Mechanical properties of glass

\begin{tabular}{|l|l|}
\hline Young's modulus $^{\mathrm{a}}$ & $E=68 \mathrm{GPa}$ \\
Poisson's coefficient $^{\mathrm{a}}$ & $\nu=0.19$ \\
Modified elastic modulus (see eq. (7)) & $E^{*}=35.3 \mathrm{GPa}$ \\
Hamaker coefficient & $A=6.5 \times 10^{-20} \mathrm{~J}$ \\
\hline Minimum distance between atoms & $r_{0}=0.3 \mathrm{~nm}$ \\
\hline
\end{tabular}

${ }^{a}$ source: www.matweb.com for $\mathrm{SiO}_{2} 96 \%$

\subsection{Approximation of nano-sized spheres}

This section is focused on the second asymptotic solution of eq. (14) which represents the case of nano-sized spheres.

Another way to normalize eq. (14) is to divide it by a $\lambda r_{0} R$ term:

$$
\left(\frac{a_{\infty}}{\sqrt[3]{\lambda r_{0} R}}\right)^{3}-\left(\frac{R}{R_{c}}\right)^{1 / 3}\left(\frac{a_{\infty}}{\sqrt[3]{\lambda r_{0} R}}\right)^{2}-1=0
$$

So for $R / R_{c}<<a_{\infty} / \sqrt[3]{\lambda r_{0} R}$, eq. (19) becomes:

$$
\left(\frac{a_{\infty}}{\sqrt[3]{\lambda r_{0} R}}\right)^{3} \approx 1
$$

Anf for $R<<R_{c}$, the contact radius and adhesion force become:

$$
\begin{aligned}
& \tilde{a}_{d v d W}=\sqrt[3]{\lambda r_{0} R}=\sqrt[3]{\frac{A R^{2}}{8 r_{0}^{2} E^{*}}}, \\
& \tilde{F}_{d v d W}=-\frac{A R}{6 r_{0}^{2}}=F_{s p} .
\end{aligned}
$$

In the case of glass $\left(R_{c}=0.5 \mu \mathrm{m}\right)$, this model matches the classical van der Waals theory at nanoscale $(R<<$ $R_{c}$ ). Moreover, the classical theory cannot predict the contact radius so the original expression (20) completes the current theory.

\subsection{Discussion}

The aim of this discussion is to estimate the error in the analytical expressions $\tilde{F}_{d v d W}$ proposed in (18) and (21) compared to an exact solution $F_{d v d W}$ of eq. (14). A coefficient $Q_{F}$ is defined as a normalized force error quotient:

$$
Q_{F}=\left|\frac{F_{d v d W}-\tilde{F}_{d v d W}}{F_{d v d W}}\right|=\left|\frac{\Delta F}{F}\right|
$$


Considering eq. (10), $F_{d v d W}$ can be written as:

$$
F_{d v d W}=\lim _{n \rightarrow \infty} F_{n}=-\frac{A\left(r_{0} R+2 a_{d v d W}^{2}\right)}{6 r_{0}^{3}} .
$$

The estimation of the error in the analytical expressions consists in estimating the error in the force $Q_{F}$ as a function of the radius $R$ of the sphere. The limit of validity of nano and microsized spheres approximations depends on the normalized error $\epsilon$ between the analytical expression $\tilde{a}_{d v d W}$ for the contact radius and the exact contact radius $a_{d v d W}$ :

$$
\frac{a_{d v d W}}{\tilde{a}_{d v d W}}=1+\epsilon
$$

where $\epsilon$ is supposed to be negligible compared to 1 .

\subsubsection{Estimation of error in the case of micro-sized spheres}

For $R>>R_{c}$, considering the definition (24) of $\epsilon$ and the value (17) of $\tilde{a}_{d v d W}$, equation (15) can be written as:

$$
(1+\epsilon)^{3}-(1+\epsilon)^{2}-\frac{R_{c}}{R}=0 .
$$

Considering that the normalized error $\epsilon$ is negligible compared to 1, eq. (25) becomes:

$$
\epsilon \approx \frac{R_{c}}{R}<<1
$$

This result can be used to estimate $Q_{F}$ :

$$
\text { For } R>>R_{c}, \quad Q_{F} \approx 3 \frac{R_{c}}{R} .
$$

Eq. (27) gives an estimation of the error if the analytical formula (18) is used rather than a numerical solution of eq. (14). This relation can be used to determine the validity domain of eq. (18). We consider that the model (18) is valid only if the relative error $Q_{F}$ is smaller than a criterion $1 / k$. The parameter $1 / k$ represents the maximum relative error of the model (typically $1 / \mathrm{k}=10 \%$ ) within its validity domain. The validity domain is thus defined by:

$$
Q_{F} \leq 1 / k \text {. }
$$

According to eq. (27) this inequation can be expressed as a function of the sphere radius $R$ rather than the relative error $Q_{F}$ :

$$
Q_{F} \leq 1 / k \Leftrightarrow R \geq 3 k R_{c}
$$

For glass, an error in force less than $10 \%$ can be observed for $R \geq 3 \times 10 R_{c}=15 \mu \mathrm{m}$ (Fig. 8 ). So this approximation is clearly validated for micro-sized spheres.

\subsubsection{Estimation of error in the case of nano-sized spheres}

In the same way, using the nanosize approximation $R<<R_{c}$, we can show that:

$$
\epsilon \approx \frac{1}{3 \sqrt[3]{R_{c} / R}-2}<<1
$$

and thus:

$$
Q_{F} \leq 1 / k \Leftrightarrow R \leq 1 / k^{3} R_{c}
$$

For glass, an error in force less than $10 \%$ can be observed for $R \leq 1 / 10^{3} R_{c}=0.5 \mu \mathrm{m}$. For such small objects, the hypothesis $R>>r_{0}$ used for the Hamaker summation in Section 3.4 is no longer valid. This fact shows that the deformation cannot be neglected in the nanoscale either. However, the classical van der Waals expression found at nanoscale (21) gives an order of magnitude of the force. Indeed, the exact solution of equation (14) and the classical van der Waals expression (20) give the same order of magnitude for $k=1\left(\left|F_{d v d W}\right| \leq 2\left|F_{s p}\right|\right)$, i.e. for $R \leq R_{c}=0.5 \mu \mathrm{m}$. 


\section{vdW theory $\quad$ vdW theory + deformation}

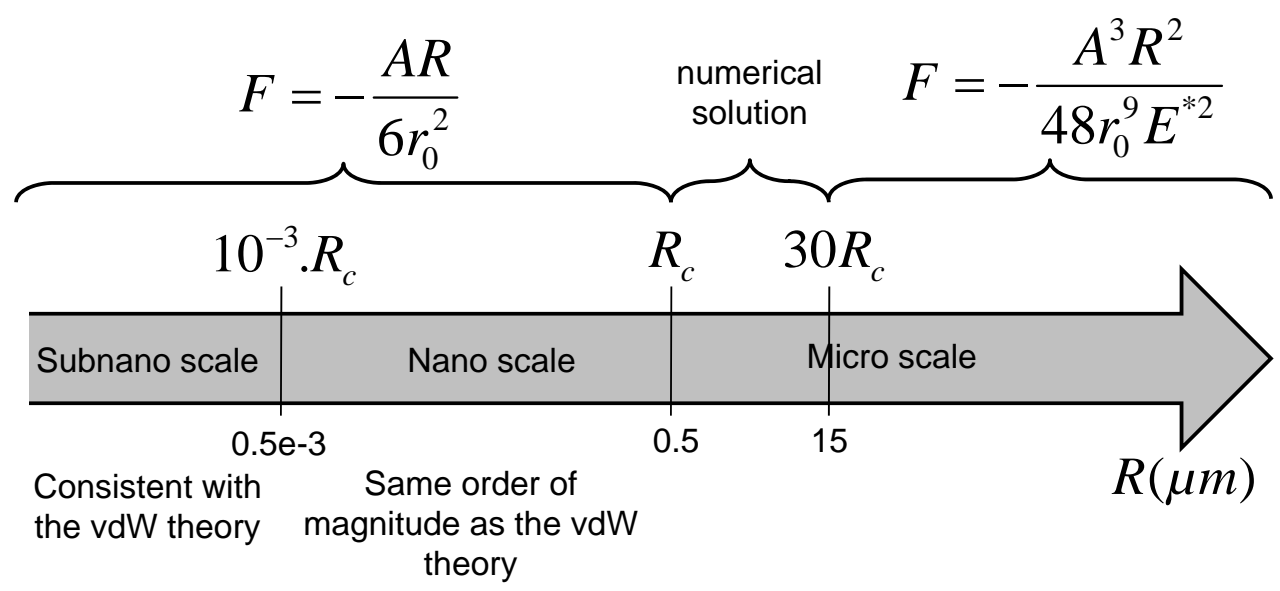

Figure 8: Adhesion models depending on sphere radius (critical radius $R_{c}$ calculated for a glass sphere).

\section{Future work}

In order to validate the proposed models, comparisons with experimental data and finite element simulation will be performed in the near future. In this way, the influence of the assumptions on deformation shape and on the rigid plane (for the integration of van der Waals forces) could be analyzed.

In a more general way, two similar studies that consider capillary condensation and electrostatic forces as the main causes of adhesion are also planned. These will help us to know which phenomenon is preponderant according to the environment characteristics and the object's material. These studies should be the way to build a simulator based on numerical integration applicable to more complex geometries. This numerical tool will be able to predict micro-objects interaction, which should be able to provide design rules in micromanipulation.

\section{Conclusion}

A new principle for adhesion forces computation has been proposed in this paper. The principle is based on the calculation of the coupling between van der Waals forces and the deformation of the object. Indeed, van der Waals forces induces a deformation that increases the contact surface, and at the same time the increased contact surface also increases the van der Waals forces itself. In order to solve this coupling problem, the proposed algorithm uses two independent models: Hertz model for the deformation and analytical expression for the van der Waals forces. In case of sphere/plane contact, analytical expressions for the deformable van der Waals forces have been proposed and their validity domains have been determined. The proposed algorithm can be extended to other more complex geometries using numerical computations.

This work has shown that the impact of the deformation of the object cannot be neglected especially in the microscale. In the nanoscale, the impact of the deformation is small and an order of magnitude of van der Waals forces can be found by neglecting the deformation.

\section{Acknowledgments}

This work is supported by the French National Project NANOROL ANR-07-ROBO-0003. Thanks to Dr Wei Dong for reviewing the manuscript.

\section{References}

[1] K. L. Johnson, K. Kendall and A. D. Roberts. Proc. R. Soc. Lond. A, 324, 301-313 (1971).

[2] B. V. Derjaguin, V. M. Muller and Yu. P. Toporov. J. Colloid Interface Sci., 53, 314-326 (1975).

[3] D. Maugis. J. Colloid Interface Sci., 150, 243-269 (1992).

[4] D. Maugis. Contact, Adhesion and Rupture of Elastic Solids. Springer (2000).

[5] M. Savia, Q. Zhou and H. N. Koivo, in: Proceedings of 2004 IEEE/RSJ International Conference on Intelligent Robots and Systems, Sendai, Japan, pp. 1722-27 (2004). 
[6] F. W. DelRio, M. P. de Boer, J. A. Knapp, E. D. Reedy, P. J. Clews and M. L. Dunn. Nature Materials, 4, 629-634 (2005).

[7] P. Lambert. Capillary Forces in Microassembly, Modeling, Simulation, Experiments, and Case Study. SpringerVerlag GmbH (2007).

[8] E. Charrault, C. Gauthier, P. Marie and R. Schirrer. Langmuir, 25, 5847-54 (2009).

[9] B. Lopez-Walle, M. Gauthier and N. Chaillet. IEEE Trans. Robotics, 24, 897-902 (2008).

[10] M. Sausse Lhernould, A. Delchambre, S. Régnier and P. Lambert. Appl. Surface Sci., 253, 6203-6210 (2007).

[11] I. N. Sneddon. Inter. J. Eng. Sci., 3, 47-57 (1965).

[12] V. A. Parsegian. Van der Waals forces. A Handbook for Biologists, Chemists, Engineers, and Physicists. Cambridge University Press (2006).

[13] H. C. Hamaker. Physica, 4, 1058-1072 (1937).

[14] R. A. Sauer and S. Li. J. Nanosci. Nanotechnol., 8, 1-17 (2007). 